\title{
Baker's Cyst Filled with Hematoma at the Lower Calf
}

\author{
Moon-Jib Yoo, MD, Jae-Sung Yoo, MD, Ho-Seong Jang, MD, and Chang-Hwan Hwang, MD \\ Department of Orthopedic Surgery, Dankook University College of Medicine, Cheonan, Korea
}

\begin{abstract}
Baker's cyst is a distension of the gastrocnemius-semimembranosus bursa of the knee, which communicates with the posterior portion of the joint capsule. Baker's cyst is commonly located in the inferomedial or superficial layers of the knee joint and rarely extends laterally or proximally. Complications of Baker's cysts are dissection, rupture, pseudothrombophlebitis, leg ischemia, nerve entrapment, and compartment syndrome. However, hematomas in the Baker's cyst have not been reported in Korea. We report a rare case of hematoma in the Baker's cyst with subfascial extension into the calf. The hematoma was demonstrated by magnetic resonance imaging and removed by mass excision.
\end{abstract}

Keywords: Knee, Calf, Baker's cyst, Hematoma

Baker's cyst is a distension of the gastrocnemius-semimembranosus bursa of the knee, which communicates with the posterior portion of the joint capsule. Such a communication was found in $30 \%$ to $50 \%$ of cadaveric dissection ${ }^{1)}$. Magnetic resonance imaging (MRI) evidence of Baker's cyst is seen in $4.7 \%$ to $19 \%$ of patients with symptoms of internal knee derangement ${ }^{2)}$. Baker's cyst is commonly located in the inferomedial or superficial layers of the knee joint and rarely extends laterally or proximally ${ }^{3)}$. Although most Baker's cysts present as an asymptomatic mass occurring directly below the popliteal fossa, they may cause clinical problems such as infection, thrombophlebitis, compartment syndrome, and entrapment neuropathy ${ }^{4,5)}$. Rupture or dissection of the cyst causes extravasation of fluid into the calf, which can result in posterior compartment syndrome or infection ${ }^{5)}$. However, hematomas in Baker's cyst have not yet been reported in Korea. Here, we report a rare case of hematoma in Baker's cyst with subfascial extension into the calf that was treated success-

Received June 18, 2014; Revised August 17, 2014;

Accepted August 25, 2014

Correspondence to: Jae-Sung Yoo, MD

Department of Orthopedic Surgery, Dankook University College of Medicine, 119 Dandae-ro, Dongnam-gu, Cheonan 330-997, Korea

Tel: +82-41-550-3060, Fax: +82-41-556-3238

E-mail: osdankook@gmail.com

This is an Open Access article distributed under the terms of the Creative Commons Attribution Non-Commercial License (http://creativecommons.org/licenses/by-nc/3.0/) which permits unrestricted non-commercial use, distribution, and reproduction in any medium, provided the original work is properly cited. fully with mass excision.

\section{Case Report}

An 81-year-old man with a major complaint of painful mass at the lower calf was referred to our institution. Pain and swelling in his left lower leg started to develop 2 weeks prior to his presentation to our clinic and progressively worsened, eventually rendering him unable to walk. While he denied any trauma to that leg,

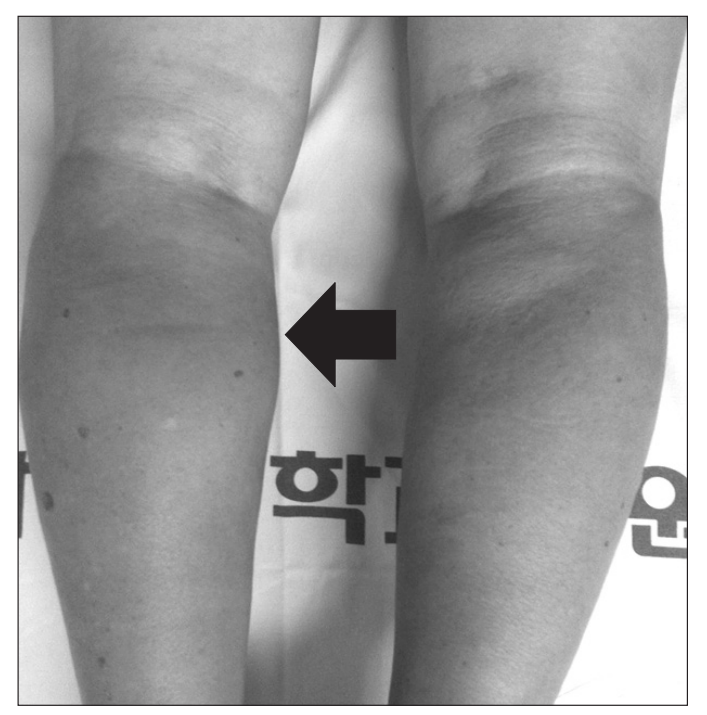

Fig. 1. The photograph of both legs shows swelling without redness in the left calf. The arrow points to the left calf. 

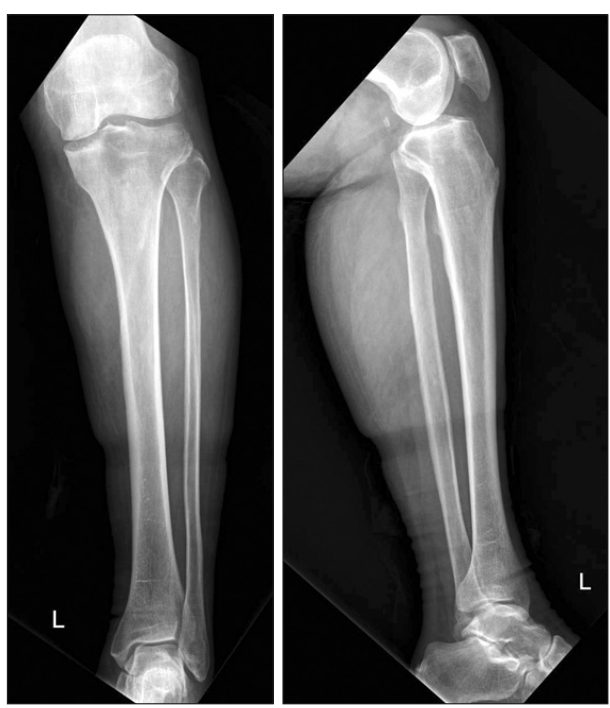

Fig. 2. Plain radiographs of the left leg show severe osteoarthritis of the knee joint and elongated soft tissue density along the medial aspect of the proximal lower leg. he had been taking aspirin $100 \mathrm{mg}$, amlodipine $5 \mathrm{mg}$, candesartan cilexetil $8 \mathrm{mg}$ per day for 7 years to control his hypertension, and had a history of several times of injections in the left knee for 1 year because of osteoarthritis.

Physical examination showed severe calf swelling with tenderness; however, no local heat or redness was observed (Fig. 1). The patient's body temperature was $36.8^{\circ} \mathrm{C}$, his dorsalis pedis artery and posterior tibial artery pulses were strong at the level of ankle and foot, and the neurological findings were normal. Knee joint line tenderness and $10^{\circ}$ of flexion contracture were observed in the left knee. The left knee score was 51 points.

The patient's blood parameters were normal: white blood cell (WBC) was 8,450, C-reactive protein (CRP) was 0.49 , and coagulation parameters and autoimmune parameters were normal. But platelet aggregation function assay was delayed. A plain X-ray of the left leg revealed Kellgren-Lawrence grade 4 osteoarthritis of the knee joint and increased soft tissue density along the medial
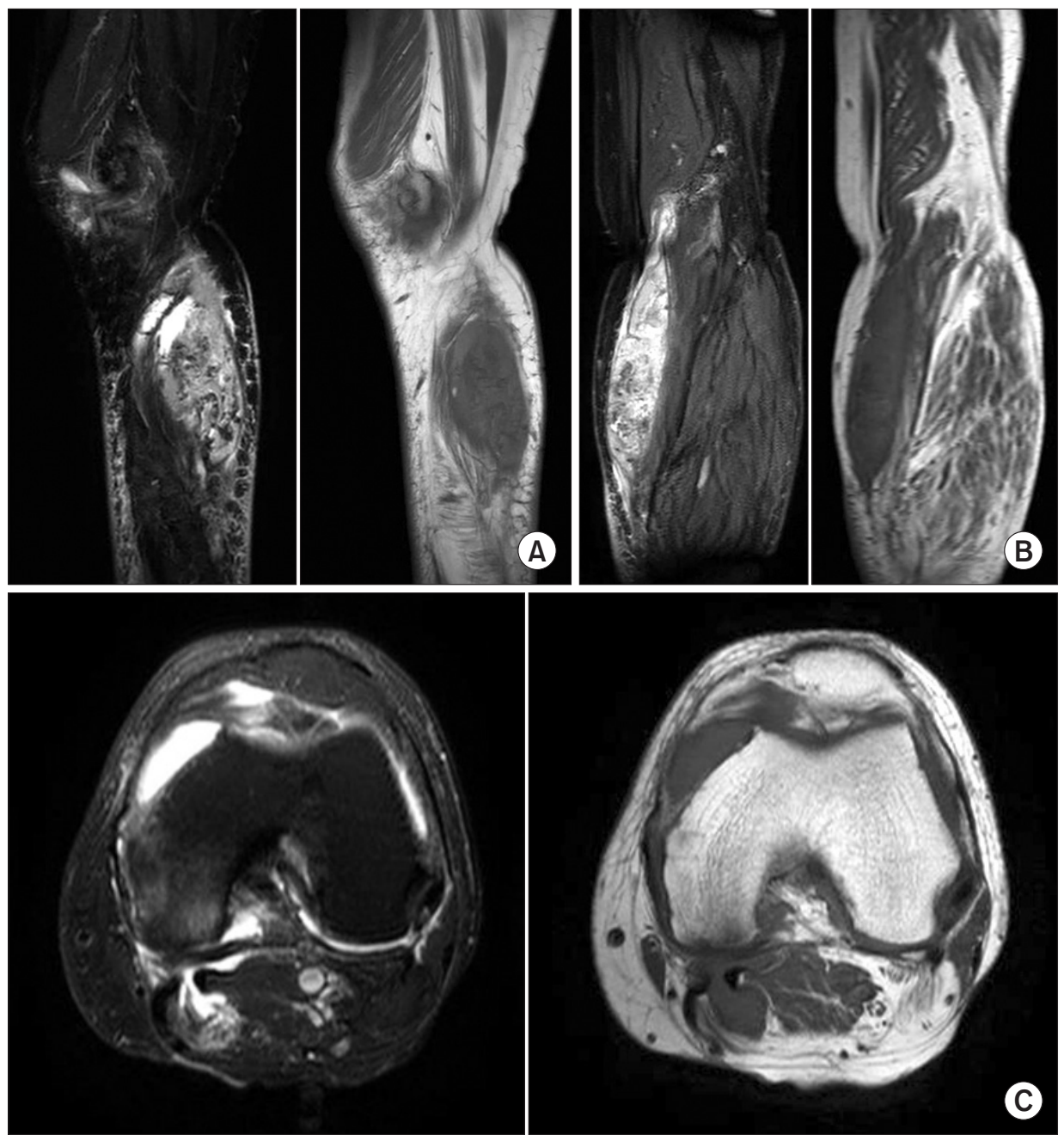

Fig. 3. Magnetic resonance imaging of the left knee. T2-weighted and T1-weighted sagittal images (A) and coronal images (B) show large cystic lesions extending distally along the subfascia in the superficial aspect of the medial gastrocnemius with muscle compression, (C) T2-weighted and T1weighted axial images. 

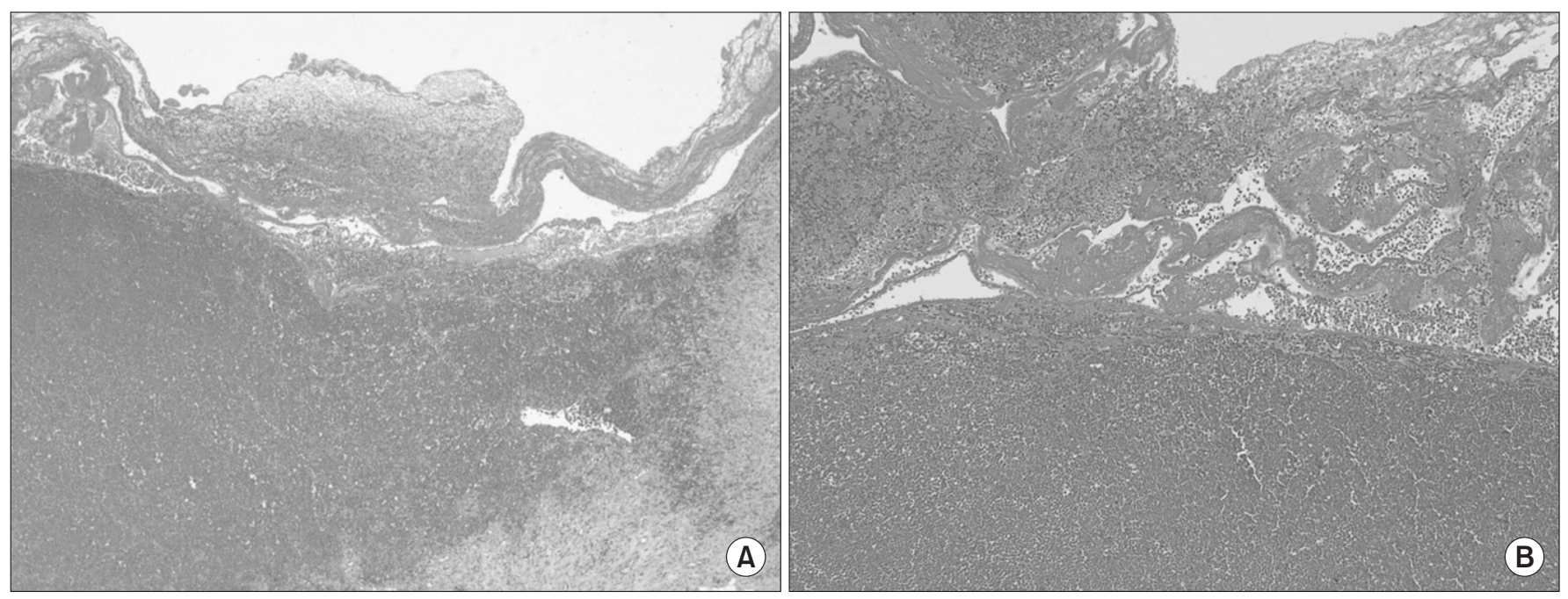

Fig. 4. Photomicrographs of the hematoma in the Baker's cyst showing macrophages consisting of phagocytosed red blood cells and hemosiderin. (A) Low-power view of the hematoma in the Baker's cyst $(\mathrm{H} \& \mathrm{E}, \times 40)$. (B) High-power view of the hematoma in the Baker's cyst (H\&E, $\times 100)$.

aspect of the proximal lower leg (Fig. 2). Computed tomography angiography (CTA) showed a large mass along the subfascial layer in the medial aspect of the gastrocnemius, and no evidence of deep venous thrombosis. MRI revealed a large mass extending distally along the subfascia in the superficial aspect of the medial gastrocnemius with muscle compression. The mass measuring $13 \times 5 \times 3 \mathrm{~cm}$ showed a low-intermediate signal intensity on T1weighted images and an intermediate-high signal intensity on T2-weighted images. These MRI findings suggested the presence of a hematoma and the inhomogeneity in signal intensity was attributed to hemorrhage within the mass (Fig. 3). Before operation, we could not rule out the possibility that MRI did not reveal any opening or stalk for direct communication between the mass and joint cavity. Therefore, an open mass excision was performed.

With the patient in the supine position under spinal anesthesia, a longitudinal skin incision was made along the medial side of the left calf. The mass was located along the subfascia in the superficial aspect of medial gastrocnemius and dark brown blood clots suggesting a hematoma was noted within the mass. During detachment from the surrounding tissues, communication between the mass and the knee joint was detected. After removal of the mass, communication with the joint cavity was repaired. The gram stain and bacterial culture of the aspirate, acid-fast, fungal staining, and culture were negative.

Histopathological examination showed that the hematoma was surrounded by synovial membrane and was composed of thick fibrous tissue; it also contained macrophages that had phagocytosed red blood cells and hemosiderin (Fig. 4). The pathologic diagnosis was hematoma in the Baker's cyst.

The patient was followed up until 2 weeks after surgery when his symptom was relieved.

\section{Discussion}

Enlargement of the gastrocnemius-semimembranosus bursa is known as a Baker's cyst ${ }^{1,4)}$. The cyst can be formed from an inter-communication between the knee joint and the bursa, and, in some cases, is associated with an intra-articular pathology of the knee, such as meniscal tears, trauma, surgery, oeteoarthritis, rheumatoid arthritis, gout, or any other forms of synovitis, ${ }^{6,7}$. Non-communicating cysts, however, often have no associated knee pathology and are thought to be primary enlargements of the gastrocnemius-semimembranosus bursa ${ }^{1,4)}$.

Although the chief complaint in most cases of Baker's cysts is an asymptomatic mass, it is common for patients to experience swelling, pain, or stiffness of the knee, often aggravated by walking. Other symptoms include bulging and tightness in the popliteal area ${ }^{1)}$.

Weakened fascia surrounding the muscle could potentially predispose to cystic dissection into the intramuscular planes. Rarely, ruptured intramuscular Baker's cyst may cause compressive neuropathy of the posterior tibial nerve. While complications associated with Baker's cysts include dissection, rupture, pseudothrombophlebitis, leg ischemia, nerve entrapment, and compartment syndrome ${ }^{8)}$, hematoma is an uncommon complication.

Ultrasonography will readily reveal a cystic structure in the popliteal fossa. Venography should only be performed if deep 
venous thrombosis is suspected. Computerized axial tomography and MRI can more effectively outline the cyst and also allow assessment of rupture of the cyst, hemorrhagic transformation, and accompanying pyomyositis or osteomyelitis ${ }^{7}$. Recently, MRI has become the preferred non-invasive diagnostic tool that provides high quality images for accurate assessment of the affected intraarticular structures and lesions, and cyst location ${ }^{9)}$.

In general, MRI findings of Baker's cysts include a low signal intensity on T1-weighted images, yet a high signal intensity on T2weighted images due to the fluid component of the Baker's cyst ${ }^{2)}$. However, the MRI of this case showed a low-intermediate signal intensity on T1-weighted images and an intermediate-high signal intensity on T2-weighted images, suggesting the presence of a hematoma. Following excision biopsy, communication between the mass and the knee joint was detected and a hematoma was found, which led to the final diagnosis of Baker's cyst.

In this report, we presented a rare case of Baker's cyst that was accompanied by hematoma causing abrupt pain and swelling in the lower extremity. This case is rare in that the MRI findings were unusual and the lower-extremity pain and swelling developed in a patient with osteoarthritis and Baker's cyst after intraarticular injections. Hematoma can be confused clinically with deep venous thrombosis or cellulitis ${ }^{10)}$. CTA and laboratory tests would be helpful for differential diagnosis.

Therefore, we believe that meticulous physical, radiological, and medical history examinations need to be carried out for exact diagnosis. We believe that this study may assist clinicians in making a proper diagnosis and administering correct treatment.

\section{Conflict of Interest}

No potential conflict of interest relevant to this article was reported.

\section{Acknowledgments}

This study was granted exemption by the Institutional Review Board of Dankook University College of Medicine.

\section{References}

1. Handy JR. Popliteal cysts in adults: a review. Semin Arthritis Rheum. 2001;31:108-18.

2. Miller TT, Staron RB, Koenigsberg T, Levin TL, Feldman F. MR imaging of Baker cysts: association with internal derangement, effusion, and degenerative arthropathy. Radiology. 1996;201:247-50.

3. Steiner E, Steinbach LS, Schnarkowski P, Tirman PF, Genant HK. Ganglia and cysts around joints. Radiol Clin North Am. 1996;34:395-425,

4. Dash S, Bheemreddy SR, Tiku ML. Posterior tibial neuropathy from ruptured Baker's cyst. Semin Arthritis Rheum. 1998;27:272-6.

5. Drees C, Lewis T, Mossad S. Baker's cyst infection: case report and review. Clin Infect Dis. 1999;29:276-8.

6. Liao ST, Chiou CS, Chang CC. Pathology associated to the Baker's cysts: a musculoskeletal ultrasound study. Clin Rheumatol. 2010;29:1043-7.

7. von Schroeder HP, Ameli FM, Piazza D, Lossing AG. Ruptured Baker's cyst causes ecchymosis of the foot. A differential clinical sign. J Bone Joint Surg Br. 1993;75:316-7.

8. Abdelrahman MH, Tubeishat S, Hammoudeh M. Proximal dissection and rupture of a popliteal cyst: a case report. Case Rep Radiol. 2012;2012:292414.

9. Sansone V, de Ponti A, Paluello GM, del Maschio A. Popliteal cysts and associated disorders of the knee. Critical review with MR imaging. Int Orthop. 1995;19:275-9.

10. Weiner SR, Fan P. Popliteal cyst involvement with gonococcal arthritis-dermatitis syndrome. Sex Transm Dis. 1983;10: 141-3. 